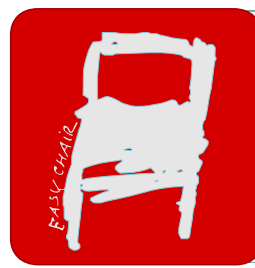

EPiC Series in Health Sciences

Volume 3, 2019, Pages 193-196

CAOS 2019. The 19th Annual Meeting of the International Society for Computer Assisted Orthopaedic Surgery

\title{
Ten to fifteen-year survival of navigation- assisted total knee arthroplasty
}

\author{
Jean-Yves Jenny, Dominique Saragaglia \\ University Hospital Strasbourg, Strasbourg, France \\ jean-yves . jenny@chru-strasbourg.fr
}

\begin{abstract}
The survival rate of navigation-assisted total knee arthroplasty when mechanical revision was considered as the end-point was $98 \%$ after 10 years and $96 \%$ after 15 years. These figures compare favorably with previously published literature about conventional implantation. Longer follow-up is required to prove superiority of any technique.
\end{abstract}

\section{Introduction}

Total knee arthroplasty (TKA) is considered a highly successful procedure. Survival rates of more than $90 \%$ after 10 years are generally reported. However, complications and revisions may still occur for many reasons, and some of them may be related to the operative technique. Computer assistance has been suggested to improve the accuracy of implantation of a TKA (Jenny 2005). Short term results are still controversial (Roberts 2015). However, few long-term results have been documented (Song 2016). The present study was designed to evaluate the long-term (more than 10 years) results of a TKA which was routinely implanted with help of a non-image-based navigation system. The 5- to 8-year of this specific TKA has already been documented (Jenny 2013).

The hypothesis of this study will be that the 10 to 15 -year survival rate of this TKA will be improved in comparison to historical papers when analyzing survival rates and knee function as evaluated by the Knee Society Score (KSS). 


\section{Material and methods}

All patients operated on between 2001 and 2004 for implantation of a navigated TKA in the two participating centers were eligible for this study. Usual demographic and peri-operative items have been recorded. All patients were prospectively followed with clinical and radiological examination. All patients were contacted after the 10-year follow-up for repeat clinical and radiological examination (KSS, Oxford knee questionnaire and knee plain X-rays). Patients who did not return were interviewed by phone call. For patients lost of follow-up, family or general practitioner was contacted to obtain relevant information about prosthesis survival. Survival curve was plotted according to Kaplan-Meier, using the occurrence of TKA revision for mechanical reason as end-point

\section{Results}

578 TKAs were implanted during the study time-frame. 537 cases had an optimal lower limb axis (HKA angle between $177^{\circ}$ and $183^{\circ}$ ) after TKA (93\%). 116 patients deceased prior to the 10 -year follow-up (20\%). Final follow-up (including death or revision) was obtained for 439 cases $(76 \%)$. Clinical status after 10 years was obtained for 341 cases (59\%) (KSS, 254 cases - Oxford questionnaire, 299 cases - radiologic evaluation, 197 cases). 10 prosthetic revisions were performed for mechanical reasons during the follow-up time (2\%). The global 10 -year survival rate was $95.5 \%$, and it decreased to $91.5 \%$ at 15 years. The 10 -year survival rate for mechanical revision only was $98.0 \%$, and decreased to $96.2 \%$ at 15 years (figure 1). The mean KSS at the last follow-up was 188 points, and the mean Oxford score was 55 points. No component was considered loose at the final radiographic evaluation. No polyethylene wear was detected at the final radiographic evaluation. No difference was observed between the two participating centers for any baseline or follow-up data. evaluation.

\section{Discussion}

The present study represents the longer follow-up of navigated TKAs published in the literature. This study confirms our initial hypothesis, namely quite satisfactory results of navigated implanted TKA after more than 10 years. Navigation, whose precision is no longer to be demonstrated, probably contributed to the quality of the results. A more consistent anatomical reconstruction and ligamentous balance of the knee should lead to more consistent survival of the TKA. Other authors did observe similar results (Baumbach 2016). However, superiority of navigated TKA in comparison to conventional implanted TKA is difficult to prove because of the subtle differences expected in mostly underpowered studies (D'Amato 2018). Longer term follow-up may be required. 
Figure 1: Survival rate - mechanical revision

\section{Survival rate - mechanical revision}

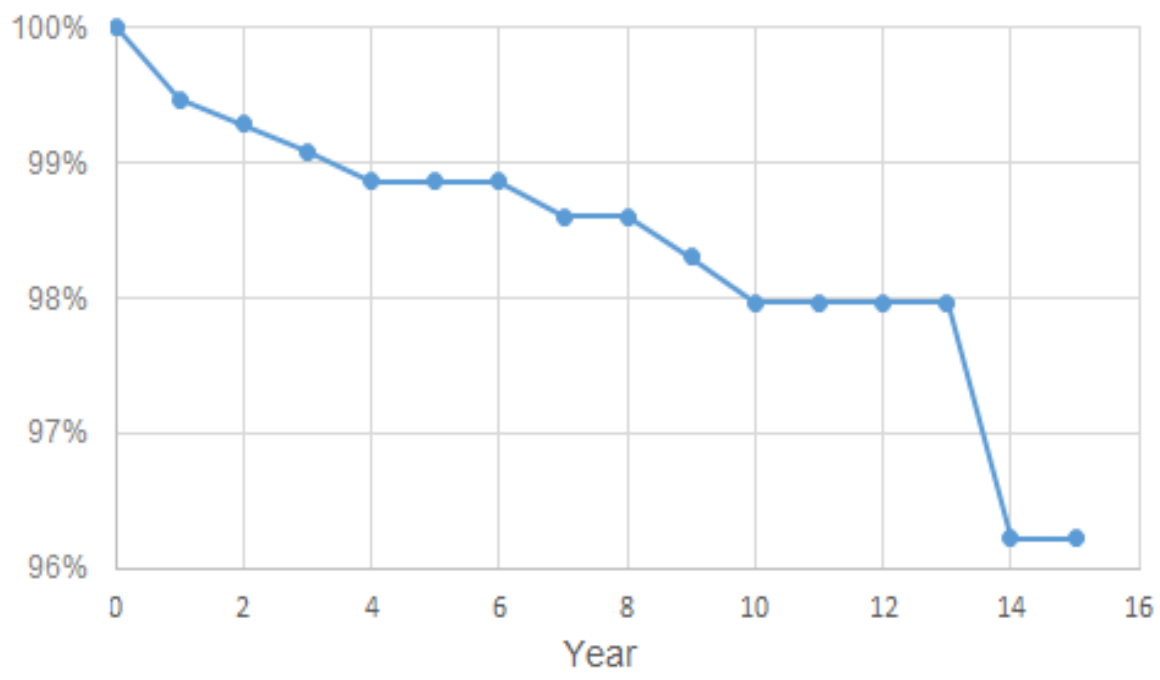

\section{References}

1. Baumbach JA, Willburger R, Haaker R, Dittrich M, Kohler S, 10-year survival of navigated versus conventional TKAs: a retrospective study, Orthopedics, 39(3 Suppl), pp: S72-76, 2016.

2. Jenny JY, Miehlke R, Saragaglia D, Geyer R, Mercier N, Schoenahl JY, Thiel B, Single-radius, multidirectional total knee replacement, Knee Surg Sports Traumatol Arthrosc, 21, pp: 2764-2769, 2013.

3. Jenny JY, Clemens U, Kohler S, Kiefer H, Konermann W, Miehlke RK, Consistency of implantation of a total knee arthroplasty with a non-image-based navigation system: a case-control study of 235 cases compared with 235 conventionally implanted prostheses, J Arthroplasty, 20(7), pp: 832-839, 2005.

4. Roberts TD, Clatworthy MG, Frampton CM, Young SW, Does computer assisted navigation improve functional outcomes and implant survivability after total knee arthroplasty?, J Arthroplasty, 30(9 Suppl), pp: 59-63, 2015.

5. Song EK, Agrawal PR, Kim SK, Seo HY, Seon JK, A randomized controlled clinical and radiological trial about outcomes of navigation-assisted TKA compared to conventional TKA: long-term follow-up, Knee Surg Sports Traumatol Arthrosc, 24(11), pp: 3381-3386, 2016.

6. D'Amato M, Ensini A, Leardini A, Barbadoro P, Illuminati A, Belvedere C. Conventional versus computer-assisted surgery in total knee arthroplasty: comparison at ten years follow-up. Int Orthop. 2018 Sep 8. doi: 10.1007/s00264-018-4114-5. [Epub ahead of print]. 


\section{Disclosure}

Jean-Yves JENNY receives royalties from AESCULAP, is a paid consultant for Exactech, is an unpaid consultant for FH Orthopedics, is member of the board of the CAOS-International Society, of the International Society for Technology in Arthroplasty and of the French Society for Hip and Knee Surgery. Dominique SARAGAGLIA receives royalties from AESCULAP 